IMSc-96/16; hep-th/9608170

\title{
Fermions in Black Hole Space-Time: Hawking Radiation and Back Reaction
}

\author{
Arundhati Dasgupta and Parthasarathi Majumdar * \\ The Institute of Mathematical Sciences, \\ CIT Campus, Madras - 600 113, India.
}

\begin{abstract}
The leading order correction to the metric of a Schwarzschild black hole, due to the backreaction of infalling fermionic matter fields, is shown to produce a shift of the event horizon such that particles that would constitute Hawking radiation at late retarded times are now trapped. Fermionic field operators associated with infalling and outgoing modes at the horizon behave canonically in the semiclassical approximation. They are, however, shown to satisfy a nontrivial exchange algebra given in terms of the backreaction, when the shift is 'quantized' by means of correspondence. The consequent exchange algebra for bilinear fermionic densities is also obtained.
\end{abstract}

Pacs nos.: 04.62.+v, 04.70.-s, 04.70.dy

Typeset using REVTEX

*E-Mail:dasgupta,partha@imsc.ernet.in 


\section{INTRODUCTION}

It is well-known that fermionic matter behaves quite differently from scalar quantum fields in black hole spacetimes. The foremost difference stems from the fact that fermionic fields are not subject to the weak energy condition [1] $T_{\mu \nu} \xi^{\mu} \xi^{\nu}>0$ on the energy-momentum tensor $T_{\mu \nu}$, where $\xi^{\lambda}$ are time-like Killing vectors. An important consequence of this difference is the absence of super-radiant scattering of fermions off charged or rotating black holes [2]. The spin statistics theorem which determines the property of the energy momentum tensor stated earlier, implies that there can be only one particle per each energy state of the fermion. It follows that the amplitude of the reflected wave cannot be enhanced by the black hole, thereby preventing the black hole from losing angular momentum or charge by fermion emission. The dissimilarity crops up again in the thermal radiation from evaporating black holes [3], where the spectral distribution from such a black hole, upon identifying its surface gravity with the Hawking temperature, is a Fermi-Dirac distribution for massless fermionic fields rather than the Planckian distribution expected for scalars.

An aspect of Hawking's seminal analysis of black hole radiation that has since received attention from diverse angles is the issue of backreaction of matter fields on the black hole geometry. In the original (asymptotic) analysis, this was considered only in an adiabatic approximation whereby the thermal radiation caused a reduction in the mass of the radiating black hole resulting in an increase of the Hawking temperature (and hence in the intensity of radiation). The consequent shrinking in the horizon size eventually leads to a complete evaporation of the black hole, leaving behind only thermal radiation, - a process which, if true, implies a breakdown of standard quantum mechanics with its law of unitary evolution. This is the notorious Information Loss Paradox. The resolution of the paradox may in principle entail modification of the principles of quantum mechanics [9], or indeed consideration

of remnant matter that retains the information (in terms of pure quantum states) that fell into the black hole [10, [11]. A less exotic approach, one which endorses standard quantum mechanics and seeks to discern aspects of the original analysis that may be subject to mod- 
ification upon inclusion of effects neglected earlier, may be another engaging possibility [4] - 6]. This more conservative viewpoint will be adopted in what follows.

According to this premise, quantum states characterizing the horizon differ sharply from the benign vacuum configuration corresponding to a fixed classical background considered in the purely semiclassical approach. The horizon is more likely a dense gas of strongly interacting particles at very high energies [4], [7] - possibly even a gas of open strings [8]. Infalling particles interact with this system according to the laws of unitary evolution, leading to a (possible) description in terms of a quantum mechanical S-matrix « involving only gravitational interactions. The large blue-shifts, close to the horizon, on the solutions of the matter field equations in the black hole geometry (which incidentally is germane to Hawking's asymptotic analysis [3]) stand to interpretation in terms of the corresponding field quanta acquiring enormous centre-of mass energies. Momentum transfers, on the other hand, are taken to be low, on account of the large angular separation expected between the most frequently colliding particles. Thus, gravitational interactions tend to dominate over all others in these kinematics.円

These interactions have been considered for scalar matter fields by Kiem et. al. [6]. The authors consider linear perturbations of the metric due to matter backreacting classically on the geometry. The modified geometry turns out to be the 'cut and paste' of two Schwarzschild geometries along the horizon (given by the Kruskal coordinate $x^{+}=0$ ) with a shift $\delta x^{-}$which is given in terms of the classical backreaction [12]. This shift, in turn, can be transcribed into a shift of the reference null Eddington-Finkelstein (EF) coordinate (defining the optimum 'time' at which a massless particle must leave $\mathcal{I}^{-}$so as to graze the horizon and just make it to $\mathcal{I}^{+}$), thereby affecting outgoing Hawking particles: the sign of the shift is such that these particles are now trapped. Consequently, the infalling and outgoing modes of the matter field operator at the horizon, which, in the absence of backreaction

\footnotetext{
${ }^{1}$ Admittedly, such kinematical arguments carry a hint of flat space intuition.
} 
are independently measurable observables, are now no longer so. Instead, their commutator is proportional to the shift of the reference null EF coordinate. A more nontrivial exchange algebra is derived between these modes, by appealing to a correspondence principle to go beyond the semiclassical approximation, by promoting the shift to an operator (albeit a bilocal one).

In this paper, we address the issue of backreaction of neutral massless fermionic matter falling on the black hole geometry. In particular, we focus on the anticommutator of the infalling and outgoing modes of the fermionic field operator at the horizon of a Schwarzschild black hole, to determine whether these modes interact gravitationally just like scalar matter. We show that the horizon undergoes a shift quite similar to the scalar case, in a linearized Einstein-Cartan formulation. Consistent propagation of fermions in the modified geometry leads to a canonical anticommutation relation between these modes in the semiclassical approximation. This leads to a canonical algebra for fermionic bilinear densities. Appealing next to the correspondence principle, a non-trivial anticommutation exchange algebra is derived for the fermionic modes. Fermionic bilinear densities satisfy an exchange algebra similar to the one found for scalar matter.

The paper is organized as follows: In the second section we briefly review the Hawking process for fermions. In section 3 we use a perturbed metric to model the effect of the back reaction of the fermion energy momentum tensor. In section 4 the effect of this is studied and the non-trivial effect on promoting the perturbation as a operator-valued quantum fluctuation is investigated. Finally in the concluding section we discuss the limitations of the method used, and the issues yet to be addressed in this approach.

\footnotetext{
${ }^{2}$ Such an algebra between two observables which are usually taken to be mutually commuting is strongly reminiscent of Complementarity in quantum mechanics. Hence the name Black Hole Complementarity for this result.
} 


\section{WEYL FERMIONS IN A SCHWARZSCHILD BLACK HOLE}

We begin with the Weyl equations for massless fermions in a Schwarzschild spacetime; the metric of the latter is given, in Kruskal-Szekeres coordinates, by

$$
d s^{2}=\frac{32(G M)^{3}}{r} e^{-r / 2 G M} d x^{+} d x^{-}-r^{2} d \Omega^{2} .
$$

A choice of tetrad components $e^{m \alpha \dot{\beta}}$ reproducing this metric is given by [2]

$$
\begin{aligned}
e^{x^{+} \dot{+}+} & =-\frac{x^{+}}{2 G M \Delta^{1 / 2}} \\
e^{x^{-\dot{-}-}} & =\frac{x^{-}}{2 G M \Delta^{1 / 2}} \\
e^{\theta \dot{-}+} & =-1 / r \quad e^{\theta \dot{+}-}=-1 / r \\
e^{\phi \dot{-}+} & =-\imath / r \sin \theta \quad e^{\phi \dot{+}-}=\imath / r \sin \theta .
\end{aligned}
$$

Here, $\Delta \equiv\left(1-\frac{2 G M}{r}\right)$. The dotted-undotted pair of indices indicate a tangent space vector as usual, with dotted (undotted) indices per se indicating chiral (antichiral) spinors of the tangent space Lorentz group. The Weyl equation is then given by,

$$
\imath \nabla^{\alpha \dot{\beta}} \psi_{\alpha}=0=\imath \nabla^{\alpha \dot{\beta}} \bar{\psi}_{\dot{\beta}}
$$

where

$$
\imath \nabla^{\alpha \dot{\beta}} \psi_{\alpha} \equiv \imath e^{m, \alpha \dot{\beta}}\left(\partial_{m} \psi_{\alpha}-\omega_{m \alpha}^{\gamma} \psi_{\gamma}\right) \text { etc. }
$$

and $\omega_{m \alpha}^{\beta}$ are the (chiral) spin connection matrices, given in terms of the tetrad components by the standard formula

$$
\omega_{m \alpha}^{\gamma} \equiv e_{\alpha \dot{\beta}}^{n} e_{n ; m}^{\gamma \dot{\beta}}
$$

Near the horizon, $\Delta \rightarrow 0$; keeping in mind that length (and time) as measured by asymptotic observers scale by the singular factor $\Delta$ in the horizon region, because of infinitely large blue-

shifts that the solutions of the dynamical equations undergo, one can rescale $\psi \rightarrow \psi / \Delta^{\frac{1}{2}}$. This reduces the pair (6) to 


$$
\begin{aligned}
& \partial_{v} \psi_{+}=0=\partial_{v} \bar{\psi}_{\dot{+}} \\
& \partial_{u} \psi_{-}=0=\partial_{u} \bar{\psi}_{\dot{-}},
\end{aligned}
$$

where $u, v$ are the Eddington-Finkelstein null coordinates. Thus, the Weyl field decomposes into 'retarded' (outgoing) and 'advanced' (incoming) solutions near the horizon, similar to the scalar field [6]

$$
\begin{gathered}
\psi^{\text {out }}=\psi_{+}(u, \Omega) \\
\psi^{\text {in }}=\psi_{-}(v, \Omega) .
\end{gathered}
$$

Since the out (in) solutions constitute a complete set of solutions to the Weyl equation on $\mathcal{I}^{+}\left(\mathcal{I}^{-}\right)$, one can match the advanced and retarded propagation of these solutions at the horizon a la' Hawking [3], leading to the reparametrization

$$
\psi^{\text {out }}(u(v), \Omega)=\psi^{\text {in }}(v, \Omega),
$$

where, $u(v)=v_{0}-4 G M \log \left(\frac{v_{0}-v}{4 G M}\right)$. The reparametrization is singular at $v_{0}$ which represents the latest reference 'time' at which an incoming wave leaves $\mathcal{I}^{-}$to get scattered to $\mathcal{I}^{+}$along the event horizon given by $u \rightarrow \infty$. For $v>v_{0}$, all incoming waves are trapped by the black hole.

Following the original treatment [3], the reparametrization above is next used to compute the (asymptotic) Bogoliubov coefficients appearing in the Bogoliubov transformations connecting the creation-annihilation operators of fields having support on the two asymptotic null infinities

$$
c_{\omega}=\int d \omega^{\prime}\left(\alpha_{\omega \omega^{\prime}} a_{\omega^{\prime}}+\beta_{\omega \omega^{\prime}} b_{-\omega^{\prime}}^{\dagger}\right),
$$

where $c, c^{\dagger}\left(a, a^{\dagger}\right)$ are the creation-annihilation operators associated with $\psi_{\text {out }}\left(\psi_{\text {in }}\right)$ on $\mathcal{I}^{+}\left(\mathcal{I}^{-}\right)$. The distinction from the scalar case now manifests as one attempts to calculate the spectral distribution of the outgoing radiation by calculating the expectation value of the number operator $c^{\dagger} c$ in the vacuum on $\mathcal{I}^{-}$; here one must recall that these operators obey 
an anticommutation algebra instead of a commutation relation. An immediate consequence is a thermal spectrum with a Fermi-Dirac distribution, upon identifying the surface gravity with the Hawking temperature [3], [2].

This analysis has obviously ignored the change in the black hole geometry induced by infalling and outgoing fermionic matter - the backreaction. In the next section we attempt to incorporate it in the semiclassical approximation: the linearized change in the black hole tetrad components due to infalling fermionic matter is determined, and re-expressed as a shift in the horizon. This is then used to determine an exchange algebra for the fermionic fields.

\section{CLASSICAL BACKREACTION FOR FERMIONS}

The dominant effect of backreaction [13] of quantum fermionic matter on the classical black hole geometry can be characterized by a linearized perturbation of the frame components: $e_{m}^{\alpha \dot{\beta}} \rightarrow e_{m}^{\alpha \dot{\beta}}+h_{m}^{\alpha \dot{\beta}}$. The linearized fluctuations $h_{m}^{\alpha \dot{\beta}}$ are related to linearized fluctuations of the Schwarzschild metric according to

$$
h_{m n}=e_{(m}^{\alpha \dot{\beta}} h_{n), \alpha \dot{\beta}}
$$

where the $e_{m}^{\alpha \dot{\beta}}$ are the Schwarzschild tetrad components given in (5). We are especially interested in the effect of infalling fermionic fields on the black hole geometry. Following Hawking's approach to black hole radiation [3], it is sufficient to restrict to waves of very high frequency near the horizon, i.e., adopt the geometrical optics approximation. For fermion fields falling on the horizon, therefore, the largest contribution to the backreaction will come from $T_{x^{+},+\dot{+}}$ and $T_{x^{-},-\dot{-}}$, the nonzero components of the energy momentum tensor in the longitudinal direction, where $T_{m}^{\alpha \dot{\beta}}$ may be taken to be (the expectation value of) the fermionic energy momentum tensor near the horizon. The other energy-momentum density components are negligible in the kinematical regime of interest.

Appealing now to the Einstein-Cartan equation 


$$
R_{m}^{\alpha \dot{\beta}}-\frac{1}{2} e_{m}^{\alpha \dot{\beta}} R=T_{m}^{\alpha \dot{\beta}}
$$

the tetrad fluctuations are seen to obey the linearized Einstein-Cartan equation (see Appendix A)

$$
\left(\nabla_{\Omega}^{2}-1\right) h_{x^{+},+\dot{+}}=k T_{x^{+},+\dot{+}}
$$

( $k$ is a constant depending upon the mass of the black hole), with the solution

$$
h_{x^{+},+\dot{+}}=\int d^{2} \Omega^{\prime} f\left(\Omega, \Omega^{\prime}\right) T_{x^{+},+\dot{+}}
$$

where, $f\left(\Omega, \Omega^{\prime}\right)$ is the Green's function of the Laplacian on the two-sphere [12]. With this modification of the geometry near the black hole horizon, consistent propagation of Weyl fermion fields, very close to the horizon, is described by the modified equations (cf. eq.s (9), 10))

$$
x^{-} \nabla_{x^{-}} \psi_{-}=0=x^{+} \nabla_{x^{+}} \psi_{+}
$$

where, $\nabla_{x^{+}} \equiv \partial_{x^{+}}-h_{x^{+} x^{+}} \partial_{x^{-}}$etc. Where $h_{x^{+} x^{+}}=e_{x^{+}}^{+\dot{+}} h_{x^{+},+\dot{+}}$. The formal solution for $\psi^{\text {out }} \equiv \psi_{+}$may be written as

$$
\psi_{+}=\psi_{+}\left(x^{-}+\int_{x_{0}^{+}}^{\infty} d y^{+} h_{x^{+} x^{+}}\left(y^{+}, \Omega\right)\right)
$$

A similar solution exists for $\psi^{i n} \equiv \psi_{-}$with a shift in the other Kruskal coordinate $x^{+}$. The important point to note in (20) above is that the integration limit excludes the interval $\left(0, x_{0}^{+}\right)$; as pointed out in [6], this region is not interesting for our purpose of estimating the effects of backreaction, since for $v<v_{0}$, all infalling waves reflect back onto $\mathcal{I}^{+}$. Backreaction effects are important only for particles that get trapped behind the horizon.

Relating the shift $\delta x^{-}$given in (20) to the affine parameter $\lambda$ of the null geodesic generator of the modified event horizon (obtained to linear order in the shift of the tetrad components), one can obtain a la' Hawking [3] a matching condition for $v>v_{0}$ between the incoming and outgoing solutions 


$$
\psi^{\text {in }}\left(v(u)+\delta v_{0}\right)=\psi^{\text {out }}(u)
$$

where,

$$
\begin{aligned}
v(u) & =v_{0}-4 G M e^{\left(v_{0}-u\right) / 4 G M} \\
\delta v_{0} & =-\int_{v_{0}}^{\infty} d v \int d \Omega f\left(\Omega, \Omega^{\prime}\right) e^{\left(v_{0}-v\right) / 4 G M} T_{v v} \quad v>v_{0}
\end{aligned}
$$

Thus, the shift in the optimum value of the EF coordinate on $\mathcal{I}^{-}$is such that fermions that would have made it to $\mathcal{I}^{+}$in the absence of backreaction, are now trapped behind the shifted horizon. A remark on the similarity of the shift $\delta v_{0}$ found here and that found in [6] for scalar matter is perhaps in order. Recall that near the horizon infalling particles are blue-shifted to enormously high energies, so that one can appeal to the geometrical optics approximation in dealing with these. Now in flat spacetime, it has been shown [14 that in this approximation (which corresponds to the eikonal approximation), fermionic and scalar cross sections become identical for electromagnetic interactions, pointing to an onshell induced supersymmetry. The similarity between the gravitational effect of fermions and scalars on the horizon seen here may be attributed to such a supersymmetry. Clearly, in the geometrical optics approximation, helicity-flip amplitudes for Weyl fermions vanish.

\section{EFFECTS OF BACKREATION}

\section{A. Semiclassical Approximation}

The semiclassical approximation, as always, considers quantum (fermionic) matter in a classical gravitational background, which in this case is a (backreaction-modified) Schwarzschild geometry. In the absence of backreaction, scalar field operators corresponding to out and in solutions of the covariant Klein-Gordon equation are known to be mutually

commuting for $v>v_{0}[6$. It is easy to see that, with the modification discussed above to the geometry, this is no longer the case: to linear order in the (c-number) shift $\delta v_{0}$, one can show that 


$$
\left[\phi^{\text {out }}(u(v), \Omega), \phi^{i n}\left(v^{\prime}, \Omega^{\prime}\right)\right]=2 \pi i \delta v_{0} \delta\left(v-v^{\prime}\right) \delta^{(2)}\left(\Omega-\Omega^{\prime}\right)
$$

Here use has been made of the canonical commutation relation for scalar fields [6]

$$
\left[\phi^{i n}\left(v_{1}, \Omega_{1}\right), \partial_{v_{2}} \phi^{i n}\left(v_{2}, \Omega_{2}\right)\right]=2 \pi i \delta\left(v_{12}\right) \delta^{(2)}\left(\Omega_{1}-\Omega_{2}\right)
$$

A similar analysis for fermionic field operators, using the canonical anticommuting relations

$$
\begin{aligned}
\left\{\psi_{\alpha}\left(v_{1}, \Omega_{1}\right), \psi_{\beta}\left(v_{2}, \Omega_{2}\right)\right\} & =0, \alpha, \beta=+,- \\
\left\{\bar{\psi}_{\dot{\mp}}\left(v_{1}, \Omega_{1}\right), \psi_{\mp}\left(v_{2}, \Omega_{2}\right)\right\} & =2 \pi i \delta\left(v_{12}\right) \delta^{(2)}\left(\Omega_{1}-\Omega_{2}\right)
\end{aligned}
$$

leads to a null result to linear order in $\delta v_{0}$ :

$$
\left\{\psi^{\text {out }}, \psi^{\text {in }}\right\}=0
$$

as one might have expected. Indeed, fermionic fields are themselves unobservable; densities constructed out of fermionic bilinears are the true observable quantities. The simplest bilinear composites constructed out of fermions are the components of the current in the tangent plane $J_{\alpha \dot{\beta}}$. The dominant components of the current ard

$$
J^{i n}(v) \equiv J_{-\dot{ }} \equiv \psi_{-}(v) \bar{\psi}_{\dot{-}}(v), J^{\text {out }}(u) \equiv \psi_{+}(u) \bar{\psi}_{\dot{+}}(u)
$$

These bilinear observables turn out also to behave canonically, i.e., $\left[J^{\text {out }}, J^{\text {in }}\right]=0$ to lowest order in $\delta v_{0}$ (see Appendix B for a sketch of the commutator calculation). This behaviour is quite in contrast to the behaviour of scalar fields in the semiclassical approximation: the infalling and outgoing modes of the fermion field appear to be completely independent, i.e., non-interacting, despite the classical shift of the horizon.

\footnotetext{
${ }^{3}$ The other components are negligible in the kinematical situation under consideration. Also, scalar bilinears vanish for chiral fermions.
} 


\section{B. Quantum Correspondence and Exchange Algebras}

With fields as operators, the energy-momentum flux operatorf $P_{v} \equiv \int_{v_{0}}^{\infty} d v T_{v v}$ has a nontrivial commutation relation with the incoming field. It generates translations along the $v$ direction.

$$
\left[P_{v}(v), \psi^{i n}\left(v^{\prime}\right)\right]=2 \pi \imath \delta^{2}\left(\Omega-\Omega^{\prime}\right) \partial_{v} \psi^{i n}\left(v^{\prime}, \Omega^{\prime}\right)
$$

Now, as seen in equation (23), the shift $\delta v_{0}$ is related to the energy momentum tensor. If we assume that this classical relation can be promoted as such to a relation between operators by means of some sort of a quantum correspondence principle [6], it can be used find the commutation relation of $\delta v_{0}$ with the incoming field

$$
\left[\delta v_{0}(\Omega), \psi^{i n}\left(\Omega^{\prime}\right)\right]=-16 \pi \imath f\left(\Omega, \Omega^{\prime}\right) e^{\left(v_{0}-v\right) / 4 G M} \partial_{v} \psi^{i n} \quad v>v_{0}
$$

This result can now be used, following Kiem et. al. [6] to determine an exchange algebra between the in and out field operators. Keeping in mind the canonical anticommutation relations obeyed by the fermion fields, this algebra (to lowest order in the backreaction) is given by

$$
\left.\psi^{\text {out }}(u, \Omega) \psi^{\text {in }}\left(v, \Omega^{\prime}\right)=-\left\{1-16 \pi \imath f\left(\Omega, \Omega^{\prime}\right) e^{(u-v) / 4 G M} \partial_{u} \partial_{v}\right\}\right) \psi^{\text {in }}\left(v, \Omega^{\prime}\right) \psi^{\text {out }}(u, \Omega)
$$

In the absence of backreaction $f\left(\Omega, \Omega^{\prime}\right)=0$, we get the standard anticommutation relation between the field operators.

Thus to the extent one can trust the procedure of promoting (23) to the level of an operator relation, fermionic field operators appear to obey the requirements of Complementarity. The point becomes clearer when one computes, within the same correspondence approach, the exchange algebra of fermionic bilinear densities; this turns out to be similar to the exchange algebra of scalar fields [6]

\footnotetext{
${ }^{4}$ The Energy Momentum tensor is not well-defined near the horizon, but the momentum flux is [䍘].
} 


$$
J^{o u t}(u, \Omega) J^{i n}\left(v, \Omega^{\prime}\right)=\left\{1-16 \pi \imath f\left(\Omega, \Omega^{\prime}\right) e^{(u-v) / 4 G M} \partial_{u} \partial_{v}\right\} J^{i n}\left(v, \Omega^{\prime}\right) J^{o u t}(u, \Omega)
$$

In other words, observables, mutually commuting in absence of backreaction, now obey a nontrivial exchange algebra (for $v>v_{0}$ ). The similarity of the exchange algebras for the fermion bilinears and scalar fields is of course, no surprise, being expected on account of the similarity in the behaviour of fermions and scalars under spacetime translations which lies at the root of the derivation of these algebras. In addition, as already mentioned, since the geometrical optics approximation is essentially being used here, and fermions and scalars behave similarly in this approximation, the only difference that may be expected is from the statistics, i.e., the fact that fermionic field operators obey a canonical anticommutator algebra. This is as obvious here as in the case of the spectral distribution of Hawking radiation.

\section{CONCLUSIONS}

In the semiclassical approximation, backreaction of matter does not seem to induce any interaction between infalling and outgoing modes of the fermion field, while for scalars such interactions do indeed appear. It is important to keep in mind however that in this analysis, tranverse gravitational interactions as well as non-gravitational forces have been ignored on the plea that they would be subdominant in this situation. Such forces could conceivably change this results somewhat [7], although a complete picture can only be expected after nonperturbative 'quantum gravity' is understood. In any case, our analysis does not indicate any serious flaw with the semiclassical approximation in the fermionic case, even though for scalars going beyond this approximation seems imperative. A deeper understanding of this difference, possibly in terms of foliations of the geometry (e.g., along lines suggested in [15]) is certainly desirable. It might be related to the diffrence mentioned at the outset, namely that fermions do not obey the weak energy condition.

As mentioned earlier, the validity of the exchange algebras derived above hinges on the assumption that the shift $\delta v_{0}$, while a shift in the optimum value of a coordinate, can be 
elevated to the level of an operator on Fock space. Clearly, as an operator, $\delta v_{0}$ is bilocal which might possibly underlie a justifiable suspicion of a violation of microcausality. In the scalar field case, Kiem et. al. introduce a third scalar field $\phi^{\text {hor }}$ to ensure that microcausality is maintained, with $\phi^{i n}$ evolving unitarily to it at the horizon from $\mathcal{I}^{-}$. It is not clear whether this is a valid procedure without a full quantum theory of gravity to back it up. We therefore adopt a deliberately ambivalent stand on this issue.

In fact, even promoting equation (23) to the level of an operator equation and thereby endowing operatorial status to a coordinate, purely by appealing to a correspondence principle, is questionable in a situation where a well-defined quantum theory is yet unknown. Usually, a correspondence principle works best in the reverse manner, i.e., in discerning the structure of a classical theory corresponding to a known quantum theory. The application of correspondence in the present context (which elevates the linearized Einstein equation to the level of an operator equation), while going beyond the purely semiclassical approximation (where gravity is classical), appears to be replete with ambiguities, again in the absence of a proper 'quantum geometry'. On the other hand, the super-Planckian energies acquired by field quanta near the horizon does seem to warrant an approach beyond the semiclassical approximation. In this context, a better approach than the one adopted here might be to follow the quantization of monopoles in gauge field theory, using the method of collective coordinates. Indeed, the horizon, as already mentioned, is modeled by a dense two dimensional gas of strongly interacting particles. Such a system, like a large nucleus, is very likely to undergo collective motion which should be amenable to exact analysis, unlike the (linearized) metric (or tetrad) fluctuations dealt with perturbatively here (the latter are analogous to Gaussian fluctuations around the ground state in the case of the nucleus). We hope to report on these issues elsewhere. 


\section{ACKNOWLEDGMENTS}

We thank S. Das, S. R. Das, P. Ramadevi and T. Sarkar for helpful discussions during the course of this work. A. D. would like to thank G. Date for bringing ref. [2] to her attention. P. M. would also like to thank the Saha Institute of Nuclear Physics for the kind hospitality extended to him during a visit during which this work was being completed, and A. P. Balachandran, A. Chatterjee, A. Ghosh, K. S. Gupta, P. Mitra and V. V. Sreedhar for illuminating discussions.

\section{APPENDIX A:}

The linear order perturbation to the tetrads is shown to satisfy equation (17) here. The

tetrad is perturbed to linear order $e_{m}^{\alpha \dot{\beta}} \rightarrow e_{m}^{\alpha \dot{\beta}}+h_{m}^{\alpha \dot{\beta}}$. Hence, to linear order in $h$, the two form,

$$
\begin{aligned}
R_{m n}^{\alpha \dot{\beta}, \gamma \dot{\delta}}= & \partial_{m} \delta \omega_{n}^{\alpha \dot{\beta}, \gamma \dot{\delta}}-\partial_{n} \delta \omega_{m}^{\alpha \dot{\beta}, \gamma \dot{\delta}}+\delta \omega_{m}^{\alpha \dot{\beta}, \alpha^{\prime} \dot{\beta}^{\prime}} \omega_{n, \alpha^{\prime} \dot{\beta}^{\prime}}^{\gamma \dot{\delta}}+\omega_{m}^{\alpha \dot{\beta}, \alpha^{\prime} \dot{\beta}^{\prime}} \delta \omega_{n, \alpha^{\prime} \dot{\beta}^{\prime}}^{\gamma \dot{\delta}} \\
& -\delta \omega_{m, \alpha^{\prime} \dot{\beta}^{\prime}}^{\gamma \dot{\delta}} \omega_{n}^{\alpha \dot{\beta}, \alpha^{\prime} \dot{\beta}^{\prime}}-\omega_{m, \alpha^{\prime} \dot{\beta}^{\prime}}^{\gamma \dot{\delta}} \delta \omega_{n}^{\alpha \dot{\beta}, \alpha^{\prime} \dot{\beta}^{\prime}} .
\end{aligned}
$$

Where $\delta \omega$ stands for the linearised spin connection. The change in the tetrad, is only along the longitudinal direction as near the horizon the energy momentum tensor of the matter field is dominant in these directions only.

$$
\delta e_{x^{+},+\dot{+}}=h_{x^{+},+\dot{+}}\left(x^{+}, x^{-}, \Omega\right), \quad \delta e_{x^{-},-\dot{-}}=h_{x,-\dot{-}}\left(x^{+}, x^{-}, \Omega\right)
$$

The changes in the spin connection very near the horizon (neglecting terms proportional to $x^{+}$and higher powers), are calculated and the relevant self dual parts are:

$$
\begin{aligned}
& \left(\delta \omega_{x^{+}}\right)_{ \pm}^{ \pm}=\mp \frac{h_{x^{+},+\dot{+}}}{\Delta^{1 / 2}} \\
& \left(\delta \omega_{x^{+}}\right)_{+}^{-}=-\frac{1}{r}\left(\partial_{\theta}+\frac{\imath}{\sin \theta} \partial_{\phi}\right) h_{x^{+},+\dot{+}}
\end{aligned}
$$

Similar equations are obtained for $\delta \omega_{x^{-}}$. The spin connections of the original metric were, 


$$
\begin{gathered}
\left(\omega_{x^{+}}\right)_{ \pm}^{ \pm}= \pm \frac{1}{2 x^{+}} \frac{4(G M)^{2}}{r^{2}}, \quad\left(\omega_{x^{-}}\right)_{ \pm}^{ \pm}= \pm \frac{1}{2 x^{-}} \frac{4(G M)^{2}}{r^{2}} \\
\omega_{\theta, \pm}^{\mp}=\mp \Delta^{1 / 2}, \quad \omega_{\phi, \pm}^{ \pm}=\mp \imath \cos \theta, \quad \omega_{\phi, \pm}^{\mp}=\imath \sin \theta \Delta^{1 / 2} .
\end{gathered}
$$

Using the above the perturbed curvature can be calculated from equation A1. The contrac-

tion with the vierbein gives $R_{m, \gamma \dot{\delta}}=e^{n, \alpha \dot{\beta}} R_{m n, \alpha \dot{\beta} \gamma \dot{\delta}}$. From Einstein-Cartan equation (16), equation (17) follows.

\section{APPENDIX B:}

The commutator of the currents are calculated to order $\delta v_{0}$.

$$
\begin{aligned}
{\left[J^{\text {out }}, J^{\text {in }}\right]=} & {\left[{\overline{\psi_{\dot{+}}}}_{\psi_{+}}, \bar{\psi}_{\dot{-}} \psi_{-}\right] } \\
= & \bar{\psi}_{\dot{+}}\left\{\psi_{+}, \bar{\psi}_{\dot{-}}\right\} \psi_{-}-\left\{\bar{\psi}_{\dot{+}}, \bar{\psi}_{\dot{-}}\right\} \psi_{+} \psi_{-} \\
& +\bar{\psi}_{\dot{+}} \bar{\psi}_{\dot{-}}\left\{\psi_{+}, \psi_{-}\right\}-\bar{\psi}_{\dot{-}}\left\{\bar{\psi}_{\dot{+}}, \psi_{-}\right\} \psi_{+}
\end{aligned}
$$

Using the anticommutators given in equations (26) and the relation of the incoming and outgoing waves,

$$
\psi^{\text {out }}=\psi_{+}(u)=\psi_{-}\left(v^{\prime}+\delta v_{0}\right), \psi^{\text {in }}=\psi_{-}(v)
$$

the above commutator is simplified to:

$$
2 \pi \imath \delta^{2}\left(\Omega^{\prime}-\Omega\right)\left[\bar{\psi}_{\dot{+}} \psi_{-}-\overline{\psi_{\dot{ }}} \psi_{+}\right]\left[\delta\left(v^{\prime}-v\right)+\delta v_{0} \partial_{v^{\prime}} \delta\left(v^{\prime}-v\right)\right]
$$

Which can be further simplified by using equation( $(\overline{B 2})$, to

$$
\begin{aligned}
{\left[J^{\text {out }}, J^{i n}\right]=} & 2 \pi \imath \delta\left(\Omega^{\prime}-\Omega\right) \delta v_{0}\left[\partial_{v^{\prime}} \delta\left(v^{\prime}-v\right)\left(\overline{\psi_{\dot{ }}}\left(v^{\prime}\right) \psi_{-}(v)-\overline{\psi_{\dot{ }}} \psi_{-}(v)\right)\right. \\
& \left.+\delta\left(v^{\prime}-v\right)\left(\partial_{v^{\prime}} \overline{\psi_{\dot{ }}}\left(v^{\prime}\right) \psi_{-}(v)-\overline{\psi_{\dot{-}}}(v) \psi_{-}\left(v^{\prime}\right)\right)\right] \\
= & 2 \pi \imath \delta\left(\Omega^{\prime}-\Omega\right) \delta v_{0} \partial_{v^{\prime}}\left[\delta\left(v^{\prime}-v\right)\left(\overline{\psi_{\dot{-}}}\left(v^{\prime}\right) \psi_{-}(v)-\overline{\psi_{\dot{ }}}(v) \psi_{-}\left(v^{\prime}\right)\right)\right] \\
= & 0
\end{aligned}
$$

Hence in the semiclassical approximation the commutator is the canonical one. 


\section{REFERENCES}

[1] S. W. Hawking and G. F. R. Ellis, The Large Scale Structure of Space-time, Cambridge University Press (1973), p. 88 et. seq.

[2] W. G. Unruh, Phys. Rev. D10, 3194 (1974).

[3] S. W. Hawking, Comm. Math. Phys. 43, 199 (1975).

[4] G. 't Hooft, Nucl. Phys. B335, 727 (1990).

[5] G. 't Hooft, The Scattering Matrix Approach for the Quantum Black Hole, grqc/9607022.

[6] Y. Kiem, H. Verlinde, E. Verlinde, Phys. Rev. D52, 7053 (1995).

[7] A. Casher, F. Englert, N. Itzhaki, S. Massar and R. Parentani, Black Hole Horizon Fluctuations, hep-th/9606106.

[8] L. Susskind, L. Thorlacius and J. Uglum, Phys. Rev. D48, 3743 (1993).

[9] S. W. Hawking, Phys. Rev. D14, 2460 (1976).

[10] Y. Aharonov, A. Casher and S. Nussinov, Phys. Lett. 191B, 741 (1987).

[11] T. Banks, M. O'Loughlin, A. Strominger, Phys. Rev. D47, 4476 (1993).

[12] T. Dray and G. 't Hooft, Nucl. Phys. 253B, 173 (1985).

[13] For a more detailed recent review, see A. Dasgupta, MSc Thesis, (IMSc-Anna University, 1996) and references therein.

[14] P. Majumdar, Induced on-shell Supersymmetry in Eikonal Particle Scattering, hepth/9509129, to appear in Phys. Rev. D.

[15] E. Keski-Vakkuri and S. D. Mathur, Quantum Gravity and Turning Points in the Semiclassical Approximation, gr-qc/9604058 (revised), to appear in Phys. Rev. D 